\title{
A!
}

This is an electronic reprint of the original article.

This reprint may differ from the original in pagination and typographic detail.

Ryytty, P.; Kaivola, M.

\section{Pulsed Standing-Wave Mirror for Neutral Atoms and Molecules}

Published in:

Physical Review Letters

DOI:

10.1103/PhysRevLett.84.5074

Published: 29/05/2000

Document Version

Publisher's PDF, also known as Version of record

Please cite the original version:

Ryytty, P., \& Kaivola, M. (2000). Pulsed Standing-Wave Mirror for Neutral Atoms and Molecules. Physical Review Letters, 84(22), 5074-5077. https://doi.org/10.1103/PhysRevLett.84.5074

This material is protected by copyright and other intellectual property rights, and duplication or sale of all or part of any of the repository collections is not permitted, except that material may be duplicated by you for your research use or educational purposes in electronic or print form. You must obtain permission for any other use. Electronic or print copies may not be offered, whether for sale or otherwise to anyone who is not an authorised user. 


\title{
Pulsed Standing-Wave Mirror for Neutral Atoms and Molecules
}

\author{
P. Ryytty and M. Kaivola \\ Department of Engineering Physics and Mathematics, Helsinki University of Technology, FIN-02015 HUT \\ Espoo, Finland
}

(Received 7 September 1999)

\begin{abstract}
Reflection of neutral atoms and molecules by a pulsed standing wave with a duration on the order of nanoseconds is studied. It is shown that, with a suitable choice of the laser parameter values, each period of the standing-wave pattern functions as an independent mirror, thus providing a novel way to manipulate large samples of neutral gas-phase particles even with a single laser pulse. At moderate field intensities, the pulsed standing-wave mirror would be directly applicable, e.g., for the manipulation of buffer-gas cooled molecules.
\end{abstract}

PACS numbers: $32.80 . \mathrm{Lg}, 33.80 .-\mathrm{b}, 42.50 . \mathrm{Vk}$

During the past few years, significant advances have been made in the production and trapping of slow gasphase molecules. Various species of ultracold alkali dimers have been produced by photoassociation in magnetooptical traps and have been subsequently captured by dipole forces [1-3]. Buffer-gas cooling followed by magnetic trapping of paramagnetic molecules in the $\mathrm{mK}$ range has been successfully implemented by Weinstein et al. [4]. Also, the use of time-varying electric fields to decelerate a beam of metastable $\mathrm{CO}$ molecules has quite recently been demonstrated [5]. The new methods for production of slow molecules open up a possibility to extend the exciting world of atom optics to also include molecular species. It is evident that this extension will drastically widen the horizon of the applications of matter-wave optics [6]. The rich internal energy-level structure of molecules, however, renders the conventional atom optical components based on light forces inapplicable to the manipulation of even the slowest molecules [7]. New approaches for the control of molecules by light fields are then needed.

Recently, several theoretical suggestions concerning the manipulation of molecules have appeared in the literature, expressing the rapidly growing interest in the topic [6,8-11]. Typically, the idea is to use pulsed lasers to increase the interaction strength between the light field and the particle. The strong electromagnetic fields attainable with pulsed lasers make it possible to utilize dipole forces to control the motion of the particles even in a far-off resonant interaction. Atom optical components based on pulsed laser fields will, therefore, not be sensitive to the internal energy-level structure of the particles and, consequently, will also allow manipulation of multilevel atoms and molecules. To date, there exist a few experimental realizations of molecular manipulation with pulsed laser fields [12,13]. Although these examples are still quite scarce, they have been able to demonstrate the great potential of the concept in providing an alternative way to realize components for atom and molecular optics.

In this paper, we study the use of a pulsed laser field for the reflection of neutral atoms and molecules. Typically, atom mirrors make use of the dipole force exerted on the particles by inhomogeneously distributed optical fields, which are tuned close to an atomic resonance frequency. In the case of blue detuning, the dipole force will repel the atoms away from the high field regions. Various field configurations based on, e.g., the use of evanescent waves have been applied to generate the repulsive potential for atomic species [14]. An efficient mirror for neutral molecules is, however, much more difficult to devise. The rich internal energy-level structure in molecules makes it necessary to apply fields tuned sufficiently far below all electronic resonances to generate the dipole force acting on the particles. In this case of quasistatic fields, however, the force exerted on a ground state molecule will always be attractive, i.e., towards the high intensity regions of the optical field. The conventional mirror geometries of atom optics cannot, therefore, be used for the reflection of ground state molecules [15]. A possible solution is to excite the molecules to Rydberg levels, which allows a repulsive dipole force to be obtained [16].

In this Letter, we propose a novel scheme based on a pulsed standing wave for realizing a simple reflector for neutral atoms and molecules that is insensitive to the internal state of the particle. We show that with a proper choice of the field intensity and pulse duration each period of the standing-wave pattern will function as an independent mirror for particles with an initial kinetic energy below the depth of the standing-wave potential. Since the node separation in a standing wave considered here is typically less than $1 \mu \mathrm{m}$, the interaction region may readily contain more than $10^{4}$ such mirrors. Each of them will provide the same dipole force to the particles and act in concert to allow manipulation of a large number of neutral atoms or molecules simultaneously with a single laser pulse. It turns out that at moderate laser intensities the pulsed standing-wave mirror (PSWM) could be used to manipulate gas-phase molecules up to velocities of several meters per second. This is enough, for example, for the control of a large volume of buffer-gas cooled molecules even with a single laser pulse. Other interesting applications could range from molecular interferometry to trapping of slow molecules in gravitational cavities. For 
the case of atoms, the PSWM can be efficiently used to reflect particles up to velocities of several tens of meters per second. This together with the increased interaction volume would make it feasible to use the PSWM even for reflection of atoms directly from a thermal beam.

The geometry of the PSWM considered in this Letter is shown in Fig. 1. The standing wave, with a cosine-squared intensity distribution in the $x$ direction, is formed in the overlap region of two counterpropagating laser beams. The period $\Lambda$ of the standing-wave pattern depends both on the wavelength $\lambda$ of the light field and on the angle $\theta$ between the beams according to the relation $\Lambda=\lambda /[2 \sin (\theta / 2)]$. The transverse profile of the laser beams is assumed to be square shaped with a time-dependent intensity. In the calculations, we assume the laser pulses to be of nanosecond duration and of moderate intensity (several tens of $\mathrm{GW} / \mathrm{cm}^{2}$ ). At the turn-on time of the laser pulse, the particles move with an initial velocity of $v_{i}$ in the direction of the normal to the wave fronts. For nonresonant light, the effects of the standing wave on the center-of-mass motion of the particles can be modeled by a position-dependent effective potential

$$
U(x, t)=U_{0}(t) \cos ^{2}\left(k_{\Lambda} x\right),
$$

where $k_{\Lambda}=\pi / \Lambda$. For linear molecules the depth of the effective potential can be approximated as $U_{0}(t)=-\alpha_{\|} E_{0}^{2}(t) / 4$, where $\alpha_{\|}$is the static polarizability of the molecule in the direction of the molecular axis and $E_{0}(t)$ is the electric field amplitude of the standing wave. This form of effective potential results when retaining the leading term in the power expansion of the eigenenergies of the pendular states and neglecting the contribution that is linear to the electric field [8]. This is a good approximation at low rotational temperatures provided that $\alpha_{\|} E_{0} /\left(4 \sqrt{B_{e} \Delta \alpha}\right) \gg 1$, where $\Delta \alpha$ is the polarizability anisotropy [6]. Since the rotational constant $B_{e}$ is inversely proportional to the reduced mass, this condition is more easily fulfilled for heavy molecules. Obviously, the approximation breaks near the node points of the standing wave. This can, however, be neglected in the calculations, since the characteristics of the PSWM are determined by the high field regions. The center-of-mass motion of the particles can now be determined by solving

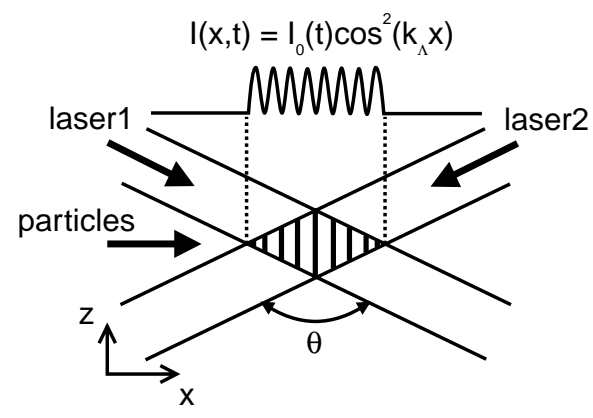

FIG. 1. The geometry of the pulsed standing-wave mirror used in the present study. the Schrödinger equation with the potential of Eq. (1) describing the effects of the standing wave.

The pulsed laser fields considered here are strong enough to transfer a momentum to the molecules several orders of magnitude larger than the recoil momentum $\hbar k_{\Lambda}$. The effects of the PSWM on the center-of-mass motion of the particles can, therefore, be determined in the quasiclassical approximation. The Schrödinger equation will then reduce to the classical equations of motion with the gradient of the effective potential representing the light forces. The final momentum distribution can be calculated by using these equations to propagate the molecules during the interaction time $T$. We assume that the initial state is evenly distributed in the position space. This allows us to limit the calculations to a single period of the effective potential. The population of a particular final momentum state is determined by counting the total number of molecules with a momentum corresponding to that state at the end of the laser pulse.

In the following, we consider the center-of-mass motion of $\mathrm{Rb}_{2}$ molecules $\left(\alpha_{\|}=135 \AA^{3}, \Delta \alpha=70 \AA^{3}\right.$ [17], $B_{e}=0.023 \mathrm{~cm}^{-1}$ [18]) in a monochromatic pulsed standing wave with a duration of $10 \mathrm{~ns}$. For this molecule $\alpha_{\|} E_{0} /\left(4 \sqrt{B_{e} \Delta \alpha}\right) \approx 20$ already at the moderate laser intensity of $13 \mathrm{GW} / \mathrm{cm}^{2}$ and, therefore, the effective potential given by Eq. (1) can be used to describe the effects of the standing wave. For the sake of clarity, we start by calculating the momentum distributions assuming a laser pulse with a fast turn-on and -off, i.e., $I_{0}(t)=I_{0}$ when $0 \leq t \leq T$ and $I_{0}(t)=0$ otherwise. In Fig. 2 we show the final momentum distribution of the molecules as a function of the initial momentum for a few values of the laser intensity. At low intensities, the standing wave does not significantly change the center-of-mass motion of the molecules and the final momentum distribution resembles the double-peaked Bessel function distribution, but with a shifted symmetry position [see Fig. 2(a)] [19,20]. When the intensity is increased [Figs. 2(b)-2(d)], the effects of the standing wave will become more pronounced. At sufficiently high intensities the molecules will go into oscillatory motion within one period of the effective potential. In this case, the shape of the final momentum distribution will become strongly dependent on the ratio of the oscillation period $T_{\text {osc }}$ to the interaction time. The most interesting results are obtained, when the oscillation period is

$$
T_{\text {osc }}=T /\left(n-\frac{1}{2}\right), \quad n=1,2, \ldots
$$

For a purely harmonic potential the molecules would then end up in a final momentum state $p_{f}=-p_{i}$ regardless of their initial momentum and $x$-coordinate value. With this choice of $T_{\text {osc }}$ the harmonic potential then acts as an elastic reflector for the molecules. The effective potential formed by a standing wave is nearly harmonic in the region around the antinodes. This allows each period of the standingwave pattern to be utilized as a molecular reflector [21]. 

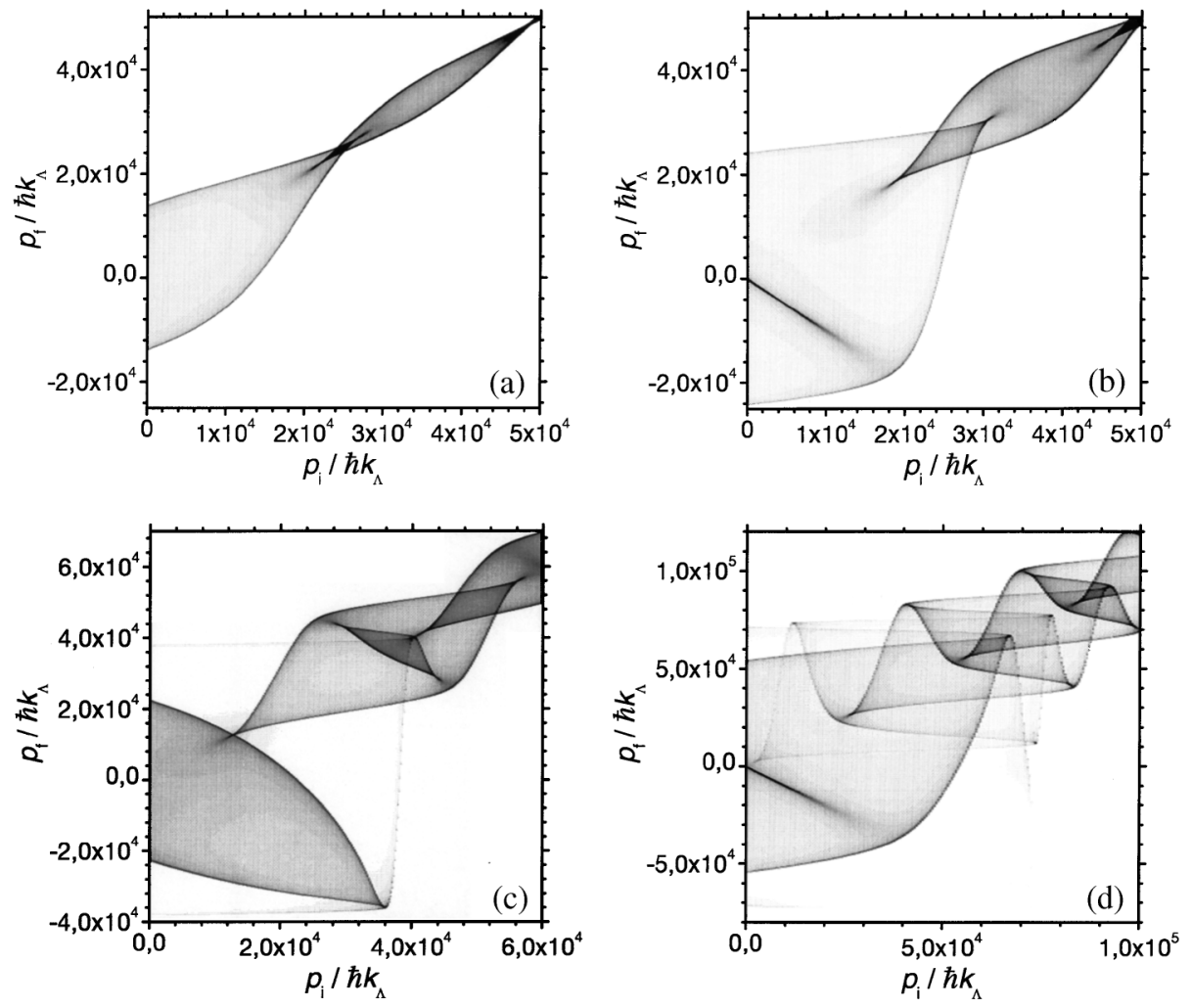

FIG. 2. Gray-scale plots of the final momentum distribution of $\mathrm{Rb}_{2}$ molecules as a function of the initial momentum. The distributions are calculated using laser pulses with a fast turn on and a duration of $10 \mathrm{~ns}$. The initial molecular distribution is chosen to be evenly distributed over the interaction region. In (a) the field intensity is $I_{0}=6 \mathrm{GW} / \mathrm{cm}^{2}\left(T_{\text {osc }}=31 \mathrm{~ns}\right)$, in (b) $I_{0}=14.5 \mathrm{GW} / \mathrm{cm}^{2}$ $\left(T_{\text {osc }}=20 \mathrm{~ns}\right)$, in (c) $I_{0}=35 \mathrm{GW} / \mathrm{cm}^{2}\left(T_{\text {osc }}=13 \mathrm{~ns}\right)$, and in (d) $I_{0}=127 \mathrm{GW} / \mathrm{cm}^{2}\left(T_{\text {osc }}=6.7 \mathrm{~ns}\right)$. An angle of $\theta=180^{\circ}$ and wavelength of $\lambda=1064 \mathrm{~nm}$ were used in all cases. Notice the dark line in (b) and (d) at $-45^{\circ}$, indicating a strong elastic reflection for molecules with $p_{i} \lesssim 12000 \hbar k_{\Lambda}$. The fraction of the nearly elastically reflected molecules is roughly $20 \%$ and $10 \%$ in (b) and (d), respectively. Also, we note that for $\lambda=10.6 \mu \mathrm{m}\left(\mathrm{CO}_{2}\right.$ laser) and $100 \mathrm{~ns}$ pulse duration the first reflection optimum would be reached at approximately $14 \mathrm{GW} / \mathrm{cm}^{2}$.

The frequency of oscillation of the molecules moving in the potential wells is to first order

$$
\omega_{\mathrm{osc}}=k_{\Lambda} v_{\max }
$$

where $v_{\max }=\sqrt{2 U_{0} / m}$ is the velocity corresponding to the depth of the effective potential $U_{0}$. Thus, with a suitable choice of the laser intensity the oscillation period can be chosen to satisfy Eq. (2). For example, in Fig. 2(b) we show the final momentum distribution for $T_{\mathrm{osc}} \approx 2 T$. Clearly, a significant fraction of the incoming molecules in the range $v_{i} T \lesssim \Lambda / 2$ (in our case $m v_{i} \lesssim 12000 \hbar k_{\Lambda}$ ) are reflected nearly elastically by the effective potential. The fraction of these molecules is limited to roughly $20 \%$ due to the anharmonic part of the effective potential. The anharmonicity effects become more pronounced at higher initial velocities, as can be seen from Fig. 2(b). This is true also for higher intensities at which the molecules will experience several oscillations in the potential well during the interaction time [see Fig. 2(d)].

In summary, to achieve maximum reflection efficiency the first reflection optimum $(n=1)$ should be used. It should also be noted that for reflection of fast molecules relatively intense laser fields are required. For example, for $v_{i} \approx 500 \mathrm{~m} / \mathrm{s}$ the required laser intensity is approximately
$1 \mathrm{TW} / \mathrm{cm}^{2}$. Such intensities can be reached by focusing a pulsed laser beam to a small spot. This will, however, lead to a reduction of the interaction volume. In addition, at high intensities the reflection efficiency will start to decrease due to multiphoton ionization.

The width of the momentum distribution of the elastically reflected molecules, $\delta p$, depends on how well relation (2) is satisfied. In a realistic case, the laser intensity varies from pulse to pulse or may have a nonuniform spatial distribution. Consequently, the oscillation period will fluctuate around the optimum value and thus the molecules will spread into a narrow band around the final momentum state $p_{f}=-p_{i}$. For example, assuming a $\pm 2 \%$ fluctuation in the intensity, the average width of the distribution of the elastically reflected molecules in Fig. 2(b) would be $\delta p \approx 200 \hbar k_{\Lambda}$. Compared with the ability of the PSWM to reflect molecules with initial momenta as high as $p_{i} \lesssim 12000 \hbar k_{\Lambda}$ this spread is, however, insignificant.

At large deviations of the oscillation period from the optimum values the molecules will spread to a wider range of momentum states, thus significantly diminishing the fraction of the elastically reflected molecules [see Fig. 2(c)]. However, the occupation of the negative momentum states can still be high as compared with the positive momentum 


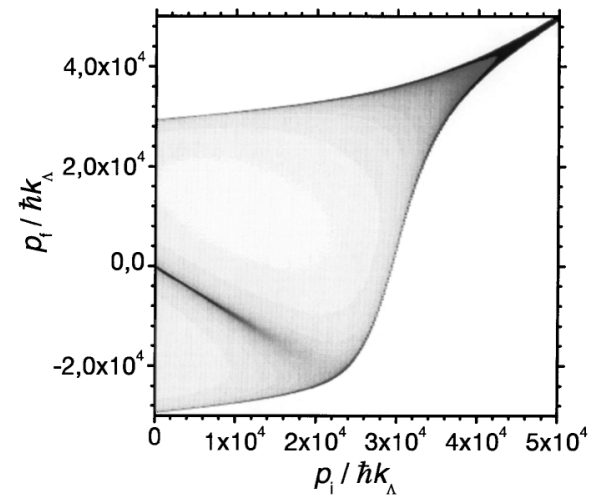

FIG. 3. Gray-scale plot of the final momentum distribution of $\mathrm{Rb}_{2}$ molecules as a function of the initial momentum in the case of a laser pulse with a Gaussian temporal profile. The $e^{-2}$ width of the profile is $10 \mathrm{~ns}$. The initial molecular distribution is chosen to be evenly distributed over the interaction region. The distribution corresponds to the first reflection optimum, i.e., to an oscillation period of $T_{\text {osc }} \approx 2 T$. The period of the standing-wave pattern is $\Lambda=532 \mathrm{~nm}$.

states. For such a nonoptimal choice of the oscillation period, the PSWM can possibly be used to inelastically reflect the incoming molecules and to modify their momentum distribution. For example, if the oscillation period is chosen to satisfy $T_{\text {osc }}=2 T /(n-1 / 2)$, the final momentum of the molecules will in the harmonic limit depend only on their initial $x$-coordinate value. Therefore, with such a choice of $T_{\text {osc }}$ a flat momentum distribution could be generated.

Finally, we show that the essential characteristics of the PSWM are not significantly altered when using more realistic laser pulses with a smooth temporal profile. For such pulses, the oscillation period will not be constant even in the case of a purely harmonic potential. In addition, the maximum momentum that can be transferred to the molecules increases more slowly as a function of the field intensity than is the case for the fast turn-on of the field [22]. These effects can, however, be compensated for by a suitable choice of the parameter values and will not cause any significant degradation of the reflection properties of the PSWM. For example, in Fig. 3, we show the final momentum distribution of the molecules as a function of the initial momentum when a laser pulse with a Gaussian temporal profile with an $e^{-2}$ width of $10 \mathrm{~ns}$ is applied. The distribution corresponds to the first reflection optimum, i.e., $n=1$ in Eq. (2). It is clearly seen that the shape of the final momentum distribution at the initial velocities of $v_{i} T \lesssim \Lambda / 2$ is similar to that in Fig. 2(b). At higher initial velocities the shape of the laser pulse will have a more significant effect on the momentum distributions.

The results of our calculations show that the PSWM could be used to efficiently manipulate large volumes of slow molecules even with a single laser pulse. With a suitable choice of the field intensity and pulse duration, the molecules can be reflected nearly elastically or with a tai- lored momentum distribution. This opens up a possibility to use the PSWM to control the motion of particles in various applications of atom and molecular physics. It could be applied, for example, to steer and image molecular beams, separate molecular species, and to form pulsed molecular beams [6]. Evidently, interest in such a component will increase in the future concurrently with the growing interest in molecular optics.

We gratefully acknowledge financial support from the Academy of Finland and from the national Finnish Graduate School of Modern Optics and Photonics. We thank Professor Juha Javanainen for a careful reading of the manuscript. P.R. gratefully acknowledges a grant from the Jenny and Antti Wihuri Fund.

[1] A. Fioretti et al., Phys. Rev. Lett. 80, 4402 (1998).

[2] T. Takekoshi, B. M. Patterson, and R. J. Knize, Phys. Rev. Lett. 81, 5105 (1998).

[3] A. N. Nikolov et al., Phys. Rev. Lett. 82, 703 (1999).

[4] J. D. Weinstein et al., J. Chem. Phys. 109, 2656 (1998); Nature (London) 395, 148 (1998).

[5] H. L. Bethlem, G. Berden, and G. Meijer, Phys. Rev. Lett. 83, 1558 (1999).

[6] T. Seideman, J. Chem. Phys. 107, 10420 (1997); Phys. Rev. A 56, R17 (1997).

[7] Microfabricated components have been successfully applied to manipulate both atoms and molecules; M.S. Chapman et al., Phys. Rev. Lett. 74, 4783 (1995).

[8] B. Friedrich and D. Herschbach, Phys. Rev. Lett. 74, 4623 (1995).

[9] J. T. Bahns, W. C. Stwalley, and P. L. Gould, J. Chem. Phys. 104, 9689 (1996).

[10] T. Seideman and V. Kharchenko, J. Chem. Phys. 108, 6272 (1998).

[11] J. Karczmarek, J. Wright, P. Corkum, and M. Ivanov, Phys. Rev. Lett. 82, 3420 (1999).

[12] H. Stapelfeldt, H. Sakai, E. Constant, and P. B. Corkum, Phys. Rev. Lett. 79, 2787 (1997).

[13] H. Sakai et al., Phys. Rev. A 57, 2794 (1998).

[14] C. S. Adams, M. Sigel, and J. Mlynek, Phys. Rep. 240, 143 (1994).

[15] In the quantum regime, mirrors based on, e.g., Bragg diffraction [P. J. Martin, B. G. Oldaker, A. H. Miklich, and D. E. Pritchard, Phys. Rev. Lett. 60, 515 (1988)] might be suitable also for molecules.

[16] T. Seideman, J. Chem. Phys. 111, 4397 (1999).

[17] M. Urban and A.J. Sadlej, J. Chem. Phys. 103, 9692 (1995).

[18] A. A. Radzig and B. M. Smirnov, Reference Data on Atoms, Molecules, and Ions (Springer, Berlin, 1985).

[19] R. J. Cook and A.F. Bernhardt, Phys. Rev. A 18, 2533 (1978).

[20] E. Arimondo, A. Bambini, and S. Stenholm, Phys. Rev. A 24, 898 (1981).

[21] Similar results can also be obtained for the diffraction of atoms by a standing wave; see, e.g., the work by Yu. B. Ovchinnikov et al. [Phys. Rev. Lett. 83, 284 (1999)].

[22] A.P. Kazantsev, G. A. Ryabenko, G. I. Surdutovich, and V. P. Yakovlev, Phys. Rep. 129, 77 (1985). 